\title{
LA DÉTERMINATION DANS LA PRATIQUE DES DIAMĖTRES VARIABLES QUI RENDENT MINIMUM LE POIDS D'UNE CONDUITE FORCÉE ÉTANT
LE DÉBIT ET LA PERTE DE CHARGE
}

\author{
Ugo BELLOMETTI \\ Ingénieur-Docteur
}

Dans une installation hydroélectrique de grande puissance et de haute chute, les conduites forcées constituent, en général, la partie la plus coûteuse de l'installation.

Parmi les diamètres que l'on peut donner à une conduite, il en est un qui est plus économique que les autres. La solution la moins coûteuse n'est pas toujours la plus économique.

Lorsqu'on augmente le diamètre d'une conduite, on en augmente le coût, mais on réduit la perte de charge. Maintenant on peut dire que cetle puissance supplémentaire valorisée en prix de vente du kw annuel se présente avantageusement vis-à-vis de l'amortissement et des intérêts des dépenses supplémentaires de l'installation du fait de la variation du diamètre.

On est donc amené à rechercher le diamètre le plus économique. Sa détermination, lorsqu'on veut lenir compte de tous les éléments économiques qui peuvent entrer en jeu est longue, difficile et surtout aléatoire, car on manque, au moment du projet, d'éléments suffisamment précis tels que nombre de $\mathrm{kw}$ de production annuelle et prix de vente de ce $\mathrm{kw}$.

La solution vraiment rationnelle devrait être choisie en partant de la loi qui détermine la perte de charge en fonction de la distance à l'origine de la conduite. Pratiquement, et pour simplifier, admettons, au contraire, que la perte de charge est une fraction de la chute totale, c'est-à-dire fixons à priori le rendement de la conduite; alors le diamètre le plus économique apparaît, comme la suite va le montrer.

Soit :

D) le diamètre constant de la conduite, en metres.

Q) le débit annuel, en mètres cubes/seconde.

H) la chute totale en mètres.

n) le nombre d'heures de fonctionnement dans l'année.

Y) la perte de charge, en mètres.

P) la puissance en cv.

L) la longueur totale d'une conduite en mètres.

C) le prix de vente de l'énergie en lire/heure.

$r$ ) le taux d'intérêts et d'amortissements.

m) le coût, en lire, pour $100 \mathrm{kgs}$ de conduite.

z) le nombre de conduites.

s) l'épaisseur moyenne de la conduite en $\mathrm{mm}$.

Le poids d'un $\mathrm{m}^{3}$ d'acier en $\mathrm{kg}_{\mathrm{g}}$ est de $8000 \mathrm{kgs} / \mathrm{m}^{3}$.

Le poids total de conduite, sans compter les accessoires : recouvrement, rivets, sera en kilogrammes: $\quad p=\frac{\Pi D s \triangle L Z}{1000}$

Supposons que l'épaisseur varie de façon continue de $O$ au maximum $s=\frac{1}{2} \frac{H D}{2 \mathrm{~K}}$ 
Si le coefficient de résistance $\mathrm{K}$ est égal à $8 \mathrm{~kg} / \mathrm{mm}^{2}$, on a $: \mathrm{s}=\frac{\mathrm{HD}}{32}$

$$
\text { et : } p=\frac{\Pi \Delta}{32000} H D^{2} L Z=0,79 H D^{2} L Z
$$

et tenant compte d'une augmentation de poids de $15 \%$ pour le recouvrement et les rivets et $10 \%$ pour les trous d'hommes, ancrages, etc. $\quad P=D^{2} H L Z$

la puissance de l'installation en cy peut ètre exprimée par : $\rho=\frac{1000}{75} \mathrm{Q}(\mathrm{H}-\mathrm{y})_{\mu}$

où ụ est le rendement de l'installation et $\mathrm{Q}$ le débit moyen.

Si nous posons $y=0.75$, la précédente formule de la puissance devient : $\quad P=10 Q(H-y)$

Et si nous exprimons la perte de charge $Y$ par la formule de chézy : $\quad y=\beta \frac{Q^{2} L}{Z^{2} D^{5}}$

d'où on tire : $P=10 Q\left(H-\beta \frac{Q^{2} L}{Z^{2} D^{5}}\right)$

le gain brut annuel sera, par suite: CnP

Si nous appelons $S$ la partie de la dépense d’installation, indépendante des conduites forcées (canaux, barrages, édifices machinerie) et $\mathrm{M}$ la dépense de main-d'œuvre et de manutention pa: an, le gain net utile sera : $\bar{b}=C n P-S r-M-D H L Z m r=$

$$
10 Q H C n-S r-M-\left(\frac{10 \beta Q^{3} L}{Z^{2} D^{5}} C n+D^{2} H L 2 m r\right)
$$

Pour rendre ce gain maximum, nous rendrons minima la quantité en parenthèses, en égalant à O sa dérivée, par rapport à la variable D, c'est-à-dire :

$$
\begin{aligned}
& \frac{10 \beta Q L}{Z^{2}}\left(-\frac{5 D^{4}}{D^{10}}\right) C n+H L 2 m r \quad 2 D=0 \\
& -\frac{50 \beta Q^{3} L C n}{Z^{2} D^{6}}+2 H D L Z m r=0 \\
& 50 \beta Q^{3} L C n=2 H D^{7} L Z^{3} m r \\
& D^{7}=\frac{50 \beta Q^{3} L C n}{2 H L Z^{3} m r} \\
& D=\sqrt[7]{\frac{25 \beta Q^{3} \mathrm{Cn}}{H Z^{3} \mathrm{mr}}}
\end{aligned}
$$

Le diamètre ainsi trouvé ne pourra être retenu, si la vitesse de l'eau dans la conduite qui en résulte se trouve exagérée et incompatible avec les temps de manouvre des surpressions trop fortes ou des dépressions excesisives dans les parties hautes de la conduite.

Par suite, des vérifications nécessaires en cas d'anomalie modifieront, en conséquence, le diamètre trouvé.

Si nous ne possédons pas tous les éléments du problème, en particulier en ce qui concerne la perte de charge, on admettra comme normale une perte de charge maximum de $2 \%$ de la hauteur de chute pour les basses chutes, pouvant aller jusqu"ì 8 à $10 \%$ pour les hautes. 
En pratique, on ne donne pas une importance excessive à la détermination du diamètre le plus économique, parce qu’il n'est pas toujours possible de réaliser ce que la théorie détermine. Tout au plus, la théorie peut servir à donner une tendance et, par suite, la détermination du diamètre constant se trouve basée surtout sur l'expérience et sur les estimations précédentes relatives au rendement ei à la sécurité de la conduite.

Ayant déterminé le diamètre constant, on passe à la recherche des diamètres variables qui rendent minimum le poids d'une condaite á égalité de débit et de perte de charge.

Posons :

D) le diamètre de la conduite.

Q) le débit de la conduite.

Y) Ia perte de charge totale dans la conduite.

h) la pression en un point quelconque de la conduite.

I.) la Iongueur totale de la conduite.

K) le coefficient de risistance du fer.

s) l'ćpaisseur nominale.

Nous savons: $s=\frac{h D}{2 K} \quad y=\frac{\beta L Q^{2}}{D^{5}}$

étant posé : $\quad \beta Q^{2}=\ddot{\gamma} \quad y=\frac{\gamma L}{D^{5}}$ (1)

Si $\delta$ est le poids ispécifique du fer, le poids de la conduite est donné par :

$$
\begin{aligned}
& P=\Pi D \delta \int_{0}^{L} s d x=\frac{\Pi \delta D^{2}}{2 K} \int_{0}^{L} h d x \\
& a D^{2} \int_{0}^{L} h d x
\end{aligned}
$$

où $a=\frac{\Pi \delta}{2 K} \quad$ est la pression en fonction de la distance $x$ de lorigine.

Si nous appelons Do le diamètre variable d'une conduite de longueur Xo, les formules 1 el 2 deviennent: $\quad y=\gamma \int_{0}^{x_{0} d x} \frac{d}{D_{0}^{5}}$

$$
P_{0}=a \int_{0}^{x_{0}} D_{0}^{2} h d x
$$

où on suppose $: h=h_{0}=$ etc

$$
P=a h_{0} \int_{0}^{x_{0}} D_{0}^{2} d x
$$

où Do dépend de $\mathrm{X}$.

La fonction Do qui rend minimum $P_{0}$ et satisfait en mème temps à la formule 3 , doil annuler la somme des dérivées partielles par rapport à $x$ des seconds membres de l'équation 3 et de l'équation 5.

Si nous rendons homogenes les fonctions, nous avons :

$$
\alpha h_{0} D_{0}^{2}-\frac{1}{\gamma D_{0}^{5}}=0 \quad D_{0}^{7}=\frac{\gamma}{2 K} \quad D=\sqrt[7]{\frac{\gamma}{2 h_{0}}}=\text { ect }
$$

On en lire, comme conséquence :

"Dans une portion de conduite horizontale, le diametre constant a choisir est celui qui conduit all poids minimum.

Par nécessité constructive et pour des raisons de résistance, indépendantes de la pression interne. on devra assigner à la portion la plus haute de la conduite une épaisseur minimum constante.

Cette portion, nous pouvons donc la considérer comme étant à pression constante et, d'après ce qui a été dit ci-dessu's, nous pouvons conclure :

«Dans une conduite de diamètre variable, la premièe portion amont ayant l'épaisseur minimum constante, doit etre de diamètre constant. » 
Si L est la longueur totale d'une conduite de profil déterminé, à portions rectilignes et descendantes, $S_{4}$ l'épaisseur minimum qui est assignée à la partie la plus haute, Do le diamètre constant de la conduite dans la portion, d'épaisseur $S_{i}$ et $X_{o}$ la longueur de cette portion, nous divisons la porlion restante de la conduite en $n$ ronçons de longueur égale.

On a supposé les longueurs égales pour simplifier et éviter des comp?ications qui rendraient les formules d'une application assez difficile, faisant perdre au problème la commodité que nous nous sommes proposée sans, par ailleurs, obtenir une économie ullérieure appréciable.

Chaque portion aura, par conséquent, la longueur : $1=\frac{L-x_{0}}{n}$

et si nous appelons $\left.D_{1}, \quad D\right), D_{n}$ les diamètres des portions successives, le poids de la conduite devient : $\quad P=a\left[\left(D_{0}^{2} h_{0} x_{0}+D_{1}^{2} \int_{0}^{1} h d x+D_{2}^{2} \int_{l}^{2 l} h d x+\ldots D_{n}^{2} \int_{(n-1) l}^{n b} h d x\right)\right]$

$$
P=\alpha \quad D_{0}^{2} h_{0} x_{0}+\sum_{m-1}^{m-n} D_{\eta}^{2} \int_{(m-1) !}^{m !} h d x
$$

et la perte de charge : $\quad y=\frac{y x_{0}}{D_{0}^{5}}+y b \sum_{m-1}^{m-n} \frac{1}{D_{m}^{5}}$ oll : $\quad y-\frac{\gamma x_{0}}{D_{0}^{5}}-\gamma\left(\sum_{m=1}^{m=n} \frac{1}{D_{m}^{5}}=F=0\right.$

Pour que $P$ soit minimum et, en même temps, satisfasse à l'équation 7 , on devra avoir :

$$
\begin{aligned}
& \frac{d P}{d D_{0}}+\lambda \frac{d F}{d D_{0}}=0 \\
& \frac{d P}{d D_{1}}+\lambda \frac{d F}{d D_{1}}=0 \\
& \frac{d P}{d D_{2}}+\lambda \frac{d F}{d D_{2}}=0 \\
& ------- \\
& \frac{d P}{d D_{n}}+\lambda \frac{d F}{d D_{n}}=0
\end{aligned}
$$

$\lambda$ est un coefficient qui rend la deuxième dérivée homogène à la première.

Ce système d’équation ajouté à l'équation 7 résout le problème. En prenant les dérivées parlielles, nous avons: $2 \alpha h_{0} D_{0} x_{0}+5 \lambda \gamma \frac{x_{0}}{D_{0}^{6}}=0$

$$
2 a D, \int_{0}^{l} h d x+5 \lambda y \frac{l}{D_{y}^{6}}=0
$$


ou :

$$
\begin{aligned}
& 2 a h_{0} D_{0}^{7}=-5 y \lambda \\
& \frac{2 a D_{1}^{7}}{1} \int_{0}^{l} h d x=-5 y \lambda \\
& \frac{2 a Q_{n}^{7}}{1} \int_{(n-1) l}^{n d} h d x=-5 y \lambda \\
& F=0
\end{aligned}
$$

Des équations $n+\mathrm{I}$, nous tirons les expressions des diamètres. $\mathrm{D}_{1}, \quad \mathrm{D}_{2}, \quad \mathrm{I}_{n}$, en fonction 10 Do.

$$
\left.\begin{array}{l}
D_{1}=D_{0}\left(\frac{h_{0} l}{\rho_{0}^{b} h d x}\right)^{1 / 7} \\
D_{m}=D_{0}\left(\frac{h_{0} !}{\int_{(m-1) !}^{m l} h^{m}}\right)^{1 / 7} \\
D_{n}=D_{0}\left(\frac{h_{0} !}{\int_{(m-1) !}^{n t} h^{1 / 7}}\right)^{1 / 7}
\end{array}\right\}(8)
$$

Substituons, dans la première équation, les valeurs de $D_{1}, D_{2}, D_{n}$, données par l'équation 8 . nous avons :

$$
\begin{gathered}
y=\frac{y x_{0}}{D_{0}^{5}}+\frac{y l}{D_{0}^{5}} \sum_{m=1}^{m=n} \frac{1}{\left(\frac{l h_{0}}{\int_{(m-1) l}^{m l} d x}\right)^{5 / 7}} \\
\gamma=\frac{\gamma}{D_{0}^{5}} \quad\left[x_{0}+\frac{l^{2 / 7}}{h_{0}^{5 / 7}} \sum_{m=1}^{m=n}\left(\int_{(m-1) !}^{m l} h d x\right)^{5 / 7}\right]
\end{gathered}
$$

et substituons à $\mathrm{Y}$ la valeur donnée par l'équation 1, nous avons :

d'ò̀ :

$$
\begin{gathered}
\frac{y l}{D^{5}}=\frac{\gamma}{D_{0}^{5}}\left[x_{0}+\frac{l^{2 / 7}}{h_{0}^{5 / 7}} \sum_{m=1}^{m=n}\left(\int_{(m-1) l}^{m l} h d x\right)^{5 / 7}\right] \\
D_{0}^{5}=\frac{D^{5}}{L}\left[x_{0}+\frac{i^{2 / 7}}{h_{0}^{5 / 7}} \sum_{m=1}^{m \cdot n}\left(\int_{(m-1) l}^{m l} h d x\right)^{5 / 7} \mid(9)\right.
\end{gathered}
$$

et d'après 8 , substituons à $D_{0}$ sa valeur en foncion de $D$.

$$
D_{m}=\frac{D}{L^{1 / 5}}\left[x_{0}+\frac{!^{p / 7}}{h_{0}^{5 / 7}} \sum_{m=1}^{m=n}\left(\int_{(m-1) l}^{m l} h d x\right)^{5 / 7}\right]^{1 / 5} \cdot\left(\frac{l h_{0}}{\int_{(m-1) l}^{m l} h d x}\right)^{1 / 7}(10)
$$

Nous avons ainsi exprimé les diamètres de lous les tronçons en fonction des diamètres constants D) (qui, pour la dite conduite, donnent la perte de charge fixe.

Le procédé à suivre est le suivant: Le profil étant donné, on fixe à priori sur la base des conditions particulières de l'installation, l'épaisseur minimum so ; on fixe également, à priori, le Do el ensuite on trouve ho et xo. 
Alors, de l'équation 9, on tire $D_{0}$ et on répète l'opération. Dans toules les applications, nous avons constaté que la deuxième donne toujours la valeur juste de Do ou, au maximum, la différence de $1 \mathrm{~mm}$. valeur qui peut donc être employée sans crainte.

Avec l'équation 10 , nous déterminerons tous les autres diamètres.

Substituons dans l'équation 6 qui donne le poids de la conduite à Dm, la valeur donnće par l'équation 8 et à Do la valeur donnée par l'équation 6 :

$$
D_{0}^{2}=\frac{D^{2}}{L^{2 / 5}}\left[x_{0}+\frac{l^{2 / 7}}{h_{0}^{5 / 7}} \sum_{(m-1) l}^{m l}(h d x)^{5 / 7}\right]^{2 / 5}
$$

Oll a : P $\quad=a\left[D_{0}^{2} h_{0} x_{0}+\sum_{m=1}^{m \cdot n}\left(\frac{h_{0} l}{\int_{(m-1) l}^{m l} h d x}\right)^{2 / 7} \int_{(m-1) l}^{m l} h d x\right]=$

$$
\begin{aligned}
& \therefore D_{0}^{2}\left[h_{0} x_{0}+\sum_{m=1}^{m=n}\left(h_{0} l\right)^{2 / 7} \cdot\left(\int_{(m-1) l}^{m l} h d x\right)^{5 / 7}\right]= \\
& \Rightarrow \\
& \therefore \quad D_{0}^{2} h_{0}\left[x_{0}+\frac{l^{2 / 7}}{h_{0}^{5 / 7}} \sum_{m=1}^{m=n}\left(\int_{(m-1) l}^{m l} h d x\right)^{5 / 7}\right]=
\end{aligned}
$$$$
\text { (11). } P=a h_{0} \frac{D^{2}}{L^{2 / 5}}\left[x_{0}+\frac{l^{2 / 7}}{h_{0}^{5 / 7}} \sum_{m=1}^{m \times n}\left(\int_{(m-1) l}^{m l} h\right)^{5 / 7}\right]^{7 / 5}
$$

Supposons, maintenant, que le diamètre Do de la portion initiale d'épaisseur constante soit egal au diamotre D du troncon suivant. De l'equation 6 , nous tirons:

$$
P=a D_{0}^{2} h_{0} x_{0}+\sum_{m=1}^{m=n} D_{m}^{2} \int_{(m-1) t}^{m l}
$$

et de l'equation $7: \quad y=\frac{y x_{0}}{D_{1}^{5}}+y \mid \sum_{m=1}^{m * n} \frac{1}{D_{m}^{5}}$

et en dérivant :

$$
\begin{aligned}
& 2 a h_{0} x_{0} D_{1}+2 a D_{1} \int_{0}^{l} h d x+5 y \lambda\left(\frac{x_{0}}{D_{1}^{6}}+\frac{l}{D_{1}^{6}}\right)=0 \\
& 2 a D_{2} \int_{L}^{2 l} h d x+5 y \lambda \frac{l}{D_{2}^{6}}=0
\end{aligned}
$$

$$
2 a D_{2} \int_{(n-1) l}^{n l} h d x+5 \gamma \lambda \frac{l}{D_{n}^{6}}=0
$$

d'ò̀ :

$$
\begin{aligned}
& \frac{2 a D_{1}^{7}}{x_{0}+l}\left(n_{0} x_{0}+\int_{0}^{l} h d x\right)=-5 \gamma \lambda \\
& \frac{2 a D_{n}^{7}}{l} \int_{(n-1) l}^{n d} h d x=-5 \gamma \lambda
\end{aligned}
$$


en exprimant tous les diamètres en fonction de $\mathrm{D}_{1}$ :

$$
\begin{aligned}
& D_{2}=D_{1} \frac{l^{1 / 7}\left(h_{0} x_{0}+\int_{0}^{l} h d x\right)^{1 / 7}}{\left[\left(x_{0}+1\right) \int_{l}^{2 l} h d x\right]^{1 / 7}} \\
& D_{2}=D_{1} i^{1 / 7}\left[\frac{h_{0} x_{0}+\int_{0}^{b} h d x}{\left(x_{0}+1\right) \int_{(n-1) l}^{n l} h d x}\right]^{1 / 7}
\end{aligned}
$$

Substituant ces valeurs dans l'équation 13 de la perte de charge et également à la valeur donnco par l'équation I pour $\mathrm{Y}$ on a :

$$
\begin{aligned}
& y=\frac{\gamma x_{0}}{D_{1}^{5}}+\frac{\gamma l}{D^{1 / 5} 1^{5 / 7}} \cdot \frac{\left(x_{0}+l\right)^{5 / 7}}{\left(h_{0} x_{0}+\int_{0}^{h} h d x\right)^{5 / 7}} \sum_{m=1}^{m=n}\left(\int_{(m-1) l}^{m l} h d x\right)^{5 / 7}= \\
& \frac{\gamma x_{0}}{D_{1}^{5}}+\frac{\gamma}{D_{1}^{5}}\left[\frac{l^{2 / 7}\left(x_{0}+l\right)^{5 / 7}}{\left(h_{0} x_{0}+\int_{0}^{l} h d x\right)^{5 / 7}} \sum_{m=1}^{m=n}\left(\int_{(m-1) l}^{m l} h\right)^{5 / 7}\right] \\
& \frac{\gamma L}{D^{5}}=\frac{\gamma x_{0}}{D_{1}^{5}} \frac{\gamma}{D_{1}^{5}}\left[\frac{l^{2 / 7}\left(x_{0}+l\right)^{5 / 7}}{\left(h_{0} x_{0}+\int_{0}^{h} h d\right)^{5 / 7}} \sum_{m=1}^{m=n}\left(\int_{(m-1) l}^{m d x}\right)^{5 / 7}\right] \\
& D_{1}=\frac{D}{L^{1 / 5}}\left[x_{0}+\frac{l^{2 / 7}\left(x_{0}+l\right)^{5 / 7}}{\left(h_{0} x_{0}+\int_{0}^{l} h d x\right)} \sum_{m=1}^{m=n}\left(\int_{(m-1) !}^{m l}\right)^{5 / 7}\right]^{1 / 5}
\end{aligned}
$$

d'où :

$$
\begin{aligned}
& D_{m}=\frac{D l^{1 / 7}}{L^{1 / 5}}\left[x_{0}+\frac{l^{2 / 7}\left(x_{0}+l\right)^{5 / 7}}{\left(h_{0} x_{0}+\int_{0}^{l} h d x\right)^{5 / 7}} \sum_{m=1}^{m=n}\left(\int_{(m-1) l}^{m l} h\right)^{5 / 7}\right]^{1 / 5}\left[\frac{h_{0} x_{0}+\int_{0}^{l} h d x}{\left(x_{0}+l\right) \int_{(m-1) l}^{m l} h d x}\right]^{1 / 7} \\
& D_{m}=\frac{D l^{1 / 7}}{L^{1 / 5}} \cdot\left[x_{0}+\frac{l^{2 / 7}\left(x_{0}+l\right)^{5 / 7}}{\left(h_{0} x_{0}+\int_{0}^{l} h d x\right)^{5 / 7}}+l^{2 / 7} \sum_{m-1}^{m l}(h d x)^{5 / 7}\right]^{1 / 5}(14)
\end{aligned}
$$

avec laquelle nous avons risolu le probleme.

Dans ce cas, on aurait besoin de faire un premier essai fixant opportunément une valeur de D, et donc, étant donné so tirer la valeur de ho et de xo.

Tirer de la formule 14, la valeur de $\mathrm{D}_{1}$ et calculer à nouveau ho et $x$.

Au second essai, nous devons avoir, normalement, les résultats exacts.

Faisant les substitutions dans l'équation 12, le poids de la conduite devient:

$$
\begin{aligned}
P . & =a\left[D_{1}^{2} h_{0} x_{0}+\sum_{m=1}^{m=n} D_{1}^{2} l^{2 / 7}\left(\frac{n_{0} x_{0}+\int_{0}^{l} h d x}{\left(x_{0}+l\right) \int_{(m-1) l}^{m l} h d x}\right)^{2 / 7} \cdot \int_{(m-1) l}^{m l} h d x\right] \\
& =a D_{1}^{2}\left[x_{0} h_{0}+1^{2 / 7} \sum_{\substack{m=n \\
m=n}}^{l}\left(\frac{h_{0} x_{0}+\int_{0}^{l} h d x}{\left(x_{0}+l\right)}\right)^{2 / 7} \cdot \sum_{(m-1) !}^{m l}\left(\int_{(m-1) l}^{m l} h d x\right)^{5 / 7}\right]
\end{aligned}
$$


Admettons encore, que le poids d'abscisse $O$ tombe à l'intérieur du premier tronçon de longueur $\frac{\mathrm{L}}{n}$

Dans la pratique, ceci est le ca's le plus fréquent, surtout dans les conduites d'une certaine importance pour lesquelles cela vaut la peine de faire l'étude des diamètres variables.

Le poids total de la conduite devient alor's :

$$
P=a\left[D_{1}^{2} h_{0} x_{0}+D_{1}^{2} \int_{x_{0}}^{l} h d x+\sum_{m=2}^{m * n} D_{m}^{2} \int_{(m-1) l}^{m l} h d x\right]
$$

La perte de charge a, d'après l'expression habituelle, la valeur de :

$$
y=\frac{\gamma L}{D^{5}}=\frac{\gamma L}{D_{1}^{5}}+\gamma L \sum_{m=2}^{m=n} \frac{1}{D_{m}^{5}}
$$

Prenons la dérivée, comme d'habitude, nous avons :

$$
\begin{aligned}
& 2 a D_{1}\left(h_{0} x_{0}+\int_{x o}^{!} h d x\right)+\frac{5 \lambda y l}{D_{1}^{6}}=0 \\
& 2 a D_{2} \int_{b}^{2 l} h d x+\frac{5 \lambda y l}{D_{2}^{6}}=0 \\
& 2 a D_{n} \int_{(n-1) !}^{n t} n d x+\frac{5 \lambda y l}{D_{n}^{6}}=0
\end{aligned}
$$

d'où on tire: $\quad D_{2}=D_{1}\left[\frac{h_{0} x_{0}+\int_{x_{0}}^{1} h d x}{\int_{1}^{2 l} h d x}\right]^{1 / 7}$

$$
D_{m}=D_{1}\left[\frac{h_{0} x_{0}+\int_{x_{0}}^{b} h d x}{\int_{(m-1) t}^{m l} h d x}\right]^{1 / 7} \text { (16) }
$$

et en substituant dans l'équation 15 , on a :

$$
y=\frac{\gamma L}{D^{5}}=\frac{\gamma l}{D_{1}^{5}}\left[1+\frac{\sum_{m=2}^{m=n}\left(\int_{(m-1) l}^{m l} h d x\right)^{5 / 7}}{\left(h_{0} x_{0} \int_{x 0}^{l} h d x\right)^{5 / 7}}\right]
$$

d'où : $\quad D_{1}=\frac{D}{n^{1 / 5}}\left[1+\frac{\sum_{m=2}^{m * n}\left(\int_{(m-1)}^{m l} h d x\right)^{5 / 7}}{\left(h_{0} x_{0}+\int_{x 0}^{l} h d x\right)^{5 / 7}}\right]^{1 / 5}$

et donc en substituant dans l'équation 16 :

$$
D=\frac{D}{n^{1 / 5}} \cdot \frac{1}{\left(\int_{(m-1) !}^{m l} h d x\right)^{1 / 7}}\left[\left(h_{0} x_{0}+\int_{x 0}^{1} h d x\right)^{5 / 7}+\sum_{m=2}^{m * n}\left(\int_{(m-1) l}^{m l} h d x\right)^{5 / 7}\right]^{1 / 5}(17)
$$

avec laquelle nous avons encore résolu le problème.

On aura besoin, naturellement, de faire l'essai habituel pour établir la valeur de $h_{0}$ et de $x_{*}$ qui résolvent le problème.

La valeur de $s_{0}$ étant fixe. 
Dans ce cas, le poids de la conduite devient :

$$
P=\alpha\left[D_{1}^{2} h_{0} x_{0}+D_{1}^{2} \int_{x_{0}}^{l} h d x+\sum_{m=2}^{m=n} D_{m}^{2}\left(\frac{h_{0} x_{0}+\int_{x_{0}}^{l} h d x}{\int_{(m-1) l}^{m h d} h d x}\right)^{2 / 7} \int_{(m-1) l}^{m l} h d x\right]
$$

Et, d'après l'équation 16 :

$$
P=a D_{1}^{2}\left(h_{0} x_{0}+\int_{x_{0}}^{L} h d x\right)\left(1+\frac{\left(\int_{(m+1)}^{m l} h d x\right)^{5 / 7}}{\sum_{m a 2}^{m m}\left(h_{0} x_{0}+\int_{x_{0}}^{l} h d x\right)^{5 / 7}}\right)
$$

Substituons la valeur de $\mathrm{D}$ :

d'où :

$$
\begin{aligned}
& P=\frac{a D^{2}}{n^{1 / 5}}\left[\left(h_{0} x_{0}+\int_{x_{0}}^{l} h d x\right)\left(1+\frac{\left(\int_{(m-1) l}^{m l} h d x\right)^{5 / 7}}{\sum_{m=2}^{m=n}\left(h_{0} x_{0}+\int_{x_{0}}^{l} h d x\right)^{5 / 7}}\right)\right] \\
& P=\frac{a D^{2}}{n^{1 / 5}}\left[\left(h_{0} x_{0}+\int_{x_{0}}^{l} h d x\right)^{5 / 7}+\sum_{m=2}^{m=n}\left(\int_{(m-1) !}^{m l} h d x\right)^{5 / 7}\right]^{7 / 5}
\end{aligned}
$$

Par suite, les formules 10-14-17 résolvent le problème dans trois cas considérés. Quel que soit le nombre de troncons d'une conduite, et le profil, nous trouvons toujours dans une des irois hypothèses faites relativement au tronçon, une épaisseur constante. En général, nous nous trouvons toujours dans les conditions du 3 " cas.

Nous avons mirs en avant la commodité de no're étude et, à première vue, les formules trouvées semb'eraient plutòt compliquées, mais si on considère que, puisque le profil d'une conduite se compose toujours d'éléments rectilignes, les inlégrales entrant dans les formules ne sont autres que les aires de plusieurs trapèzes et les intégrales qui composent le dénominateur ne sont que la somme de ces intégrales, ce qui tacili.e et accélère la solution.

Pour établir le nombre des tronçons de diamètre constant, nous ne pouvons énoncer de règles.

Pour les conduites de longueur inférieure à 500 mètres, il n'est pas besoin de subdiviser en tronçons.

Pour les conduites de plus grande longueur, on admettra la subdivision en $2,3,4,5$, etc. trrnçons.

L'économie de poids croît de manière sensible d'une valeur à la suivante ; jusqu'à 5 et alu delà, la variation est́ moins sensible de sorte que, au delà d'une certaine limite, il n'est pas opportun do s'exeiter, car léconomie qui s'ensuivrait serait annulée, sinon transformée en dépense supplémentaire par le plus grand coût de construction.

On pouria, par exemple, être conduit à adopter un plus grand nombre de diamètres, quand pour de longs et coûteux voyages, il est à conseiller d'augineñter au maximum le poids de chaque caisse enfilant le plus grand nombre de tubes l'un dans l'autre.

Quand, en particulier, sur les hautes chutes, les diamètres calculés réclament des épaisseurs supérieures aux possibilités constructives, on pourra bire conduit à diminuer, dans la partie basse, le diamè re pour une certaine longueur, rectifiant, en conséquence, la longueur des autres tronçons.

Quand, en outre, le profil de la conduite présente d'importantes parties planes, on pourra être amené à rechifier les différentes longueurs des tronçons et leur nombre.

D'après ce qui précède, la solution de cette question comme de beaucoup d'autres, intéressant les conduites forcées, ne pourra être trouvée de manière satisfaisante sans le concours de spécialistes avancés.

Connaissant le diamètre constant $\mathrm{D}$ de la conduite, les diamètres $\mathrm{D}_{1}, \mathrm{I}_{i}, \mathrm{D}_{3}, \mathrm{I}_{4}, \mathrm{D}_{i}, \mathrm{D}_{6}$,

\begin{tabular}{|c|c|c|c|c|c|c|c|}
\hline $\begin{array}{l}\text { NUMÉRO } \\
\text { TRONÇON }\end{array}$ & $\frac{D_{1}}{\bar{D}}$ & $\frac{\mathrm{D}_{2}}{\mathrm{D}}$ & $\frac{D_{i}}{\mathrm{D}}$ & $\frac{D_{3}}{D}$ & $\frac{D_{i}}{D}$ & $\frac{D_{6}}{\mathrm{D}}$ & $\begin{array}{c}\text { GAIN } \\
\operatorname{poIDS} \%\end{array}$ \\
\hline 2 & 1,093 & 0,938 & & & & & 3,8 \\
\hline 3 & 1,162 & 0,993 & 0,923 & & & & 4,8 \\
\hline 4 & 1,210 & 1,034 & 0,936 & 0,918 & & & 5,2 \\
\hline 5 & 1,248 & 1,067 & 0,983 & 0,947 & 0,913 & & 5,43 \\
\hline 6 & 1,281 & 1,095 & 1,018 & 0,972 & 0,936 & 0,910 & 5,56 \\
\hline
\end{tabular}
que, à partir de l'origine devrait avoir la conduite divisée en tronçons égaux pour aroir une égale perte de charge et le poids minimum, sont donnés par le tableau suivant, calculé en supposant la pente uniforme et des épaisseurs variant lunéairement. 


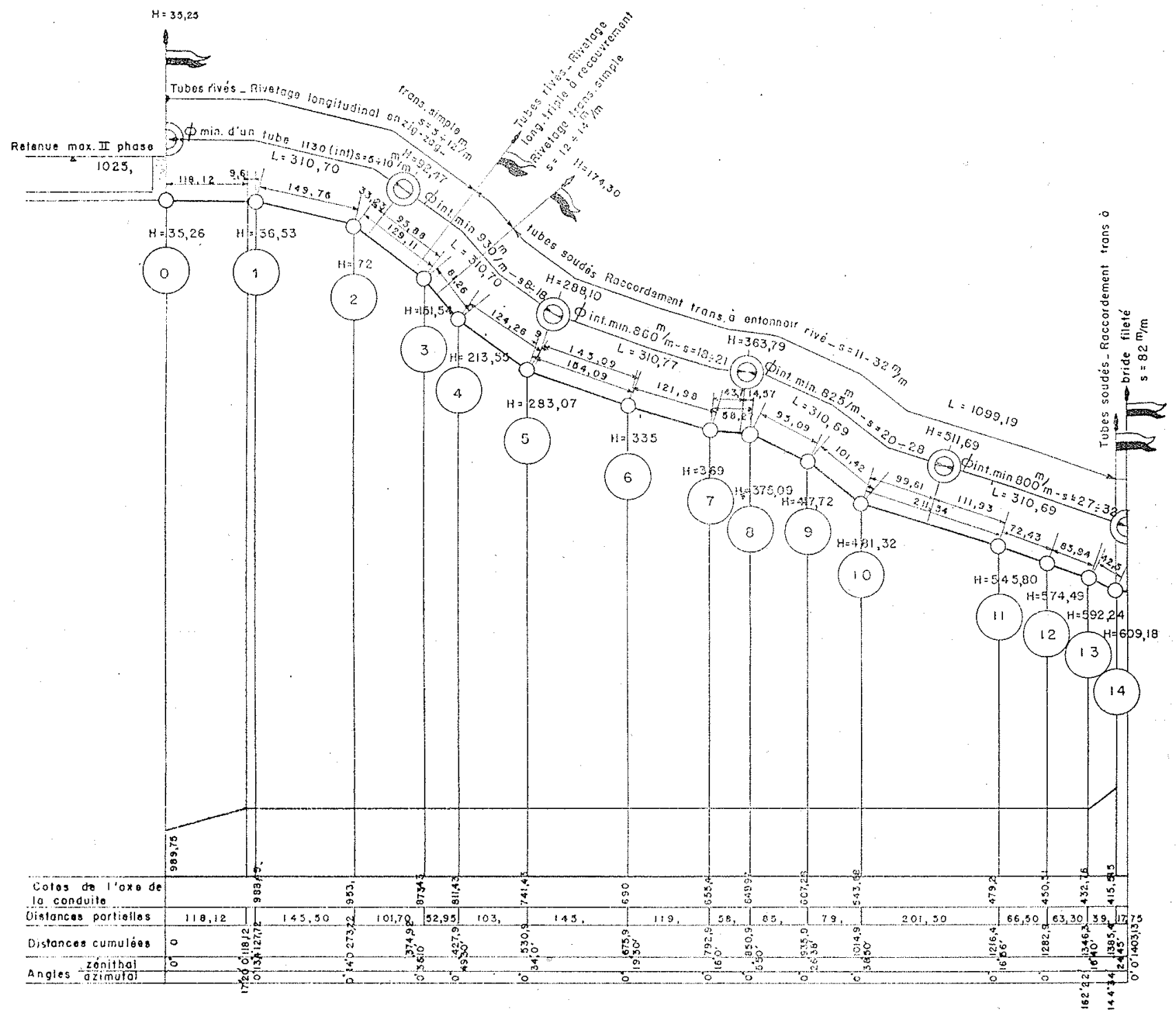

EXEMPLE NUMERIQUE DE CALCUL DES DIAMETRES VARIABLES DE POIDS MINIMUM

Nous nous référons aux données d'une importante installation qui fonctionne depuis plusieurs années et dont le profil est illustré dans la figure.

Le débit maximum esi de $2500 \mathrm{l} / \mathrm{sec}$. la longueur de $1.553 \mathrm{~m}, 48$.

Voyons comment sont calculés les différents diamètres qui composent la conduite forcée.

Subdivisons la conduite en 5 portions de longueur :

$$
\frac{1.553,48}{5}=310,70
$$

et établissons une perte de charge de $10 \%$ de la chute, soit $61 \mathrm{~m}$. Le diametre constant qui donne cette perte de charge s'obtient d'aprés la formule de Darcy:

$$
\begin{gathered}
y=0,0025 \frac{Q^{2}}{D^{5}} \cdot L \\
D=\left(0,0025 \frac{Q^{2}}{y} L\right)^{1,5}=\left(\frac{0,0025 \cdot 2,5^{2} \cdot 1553,48}{61}\right)^{1 / 5} \\
D=0,83
\end{gathered}
$$


Partant de ce diamètre, on arrive à un diamètre minimum qui conduit à une vitesse de $4 \mathrm{~m}$. $50 /$ sec. que nous considérons comme trop élevée en relation avec les caractérisliques de l'installation.

Nous augmentons donc le diamètre en réduisant la perte de charge à 7,8 \% de sorte que le diamètre résultant se trouve être de :

$$
\mathrm{D}=0 \mathrm{~m} .874
$$

que nous retenons.

Nous procédons, ainsi à la détermination des diamètres variables en parlanl de ce diamètre. En léveloppant la formule 17 trouvée précédemmeni, nous déterminons la valeur partielle qui la compose :

$$
\begin{aligned}
& \int_{0}^{L} h d x=\int_{0}^{127,72} h d x+\int_{127,72}^{277,47} h d x+\int_{277,47}^{310,70} h d x=4585+8130+2734=15449 \\
& \left(\int_{0}^{b} h d x\right)^{1 / 7}=(15449)^{1,7}=3,996 \\
& \int_{6}^{2 t} h d x=\int_{310,70}^{406,58} h d x+\int_{406,58}^{488,14} h d \int_{488,14}^{612,40} h d x=11700+14810+30870+2560=59940 \\
& \left(\int^{2 l} h d x\right)^{1 / 7}=(59940)^{1 / 7}=4,814 \\
& \left(\int_{L}^{2 l} h d x\right)^{5 / 7}=(59940)^{5 / 7}=\underline{2587} \\
& \int_{2 l}^{3 l} h d x=\int_{621,40}^{766,42} h d x+\int_{766,42}^{888,40} h d x+\int_{888,40}^{932,10} h d x=45100+43000+16225=104325 \\
& \left(\int_{2 l}^{3 L} h d x\right)^{1 / 7}=(104325)^{1 / 7}=5,211 \\
& \left(\int_{2 l}^{36} h d x\right)^{5 / 7}=(104325)^{5 / 7}=3842 \\
& \int_{31}^{41} h d x=\int_{932,10}^{946,67} h d x+\int_{946,67}^{1041,75} h d x+\int_{1041,76}^{1143,18} h d x+\int_{1143,18}^{1243,79} h d x=5460+3770 \\
& \left.\int_{31}^{41} h d x\right)^{1 / 7}=(138260)^{1 / 7}=5,425 \\
& \left(\int_{31}^{41} h d x\right)^{5 / 7}=(138260)^{5 / 7}=4698 \\
& \int_{41}^{5 l} h d x=\int_{1242,79}^{1354,72} h d x+\int_{1354,72}^{1427,35} h d x+\int_{1427,35}^{1439,09} h d x+\int_{1439,09}^{1553,48} h d x=59150+ \\
& \left(\int_{41}^{51} h d x\right)^{1 / 7}=(174530)^{1 / 7}=\underline{5,608} \\
& \left(\int_{41}^{5 l} h d x\right)^{5 / 7}=(174530)^{5 / 7}=\underline{549} \\
& \sum_{m=2}^{m * n}\left(\int_{(m-1) l}^{m l} h d x\right)=2586+3842+4698+5549=16675 \\
& \frac{D}{5^{1 / 5}}=\frac{0,874}{5^{1 / 5}}=0,6334
\end{aligned}
$$

Nous faisons un premier essai en prenant pour $D$, la valeur :

$$
0 \mathrm{~m} .874 \times 1,248=1,090
$$

$\frac{D_{1}}{\mathrm{I})}=1,248$ que donne la table précédente pour la subdivision en jo troncons. 
Pour l'épaisseur minimum so $=5 \mathrm{~mm}$, avec rivure longitudinale double et en zigzag, on a : $h=53$ à quoi correspond $x=197,22$ par suite :

$$
\begin{gathered}
h x=10450 \\
\int_{x_{0}}^{l} h d x=\int_{197,22}^{277,47} h d \int_{277,47}^{310,70} h d x=5180+2734=7914 \\
\left(h_{0} x_{0}+\int_{x_{0}}^{l} h d x\right)^{5 / 7}=(10450+7914)^{5 / 7}=1111 \\
\left.\left[\left(h_{0} x_{0}+\int_{x_{0}}^{l} h d x\right)^{5 / 7}+\sum_{m \times 2}^{m * 5} \int_{(m-1) l}^{m l} h\right)^{5 / 7}\right]^{1 / 5}=(1111+16675)=7,08 \\
D_{1}=0,6334-7,05 \cdot \frac{1}{3,966}=1,131
\end{gathered}
$$

nous répétons l'essai avec le diamètre 1,131:

$$
\begin{aligned}
& h_{c}=51,20 \quad x_{0}=139,57 \quad h_{0} x_{0}=9710 \\
& \int_{x_{0}}^{l} h d \cdot x=\int_{139,57}^{277,47} h d x+\int_{277,47}^{310,70}=5415+2734=8149 \\
& \left(h x+\int_{x}^{l} h d x\right)^{5 / 7}=(17859)^{5 / 7}=1089 \\
& \left.\left[\left(h_{0} x_{0}+\int_{x_{0}}^{l} h d x\right)^{5 / 7}+\sum_{m=2}^{m=5} \int_{(m-1) l}^{m l} h\right)^{5 / 7}\right]^{1 / 5}=(1089+16675)^{1 / 5}=7,078
\end{aligned}
$$

done :

$$
\begin{aligned}
& D_{1}=0,6334 \times 7,08 \times \frac{1}{3,966}=\underline{1,130} \\
& D_{2}=0,6334 \times 7,08 \times \frac{1}{3,966}=0,931 \\
& D_{3}=0,6334 \times 7,08 \times \frac{1}{3,966}=0,860 \\
& D_{4}=0,6334 \times 7,08 \times \frac{1}{3,966}=0,826 \\
& D_{5}=0,6334 \times 7,08 \times \frac{1}{3,966}=0,826
\end{aligned}
$$

Nous vérifions la perte de charge:

$y=0,0025 \times 2,5 \times 310,70\left(\frac{1}{1,13^{2}}+\frac{1}{0,93^{2}}+\frac{1}{0,86^{2}}+\frac{1}{0,825^{2}}+\frac{1}{0,85^{5}}\right)=4,86(0,543+1,436+2,128+2,620+3,058)=$ $47,50 \sim$

cest-ì-dire : $7,8 \%$.

Nous avons ainsi, déterminé les diamèt 